\author{
Associate Professor Yingyi HUANG, PhD \\ E-mail: hyy@qztc.edu.cn \\ TSL Business School, Quanzhou Normal University \\ Associate Professor Shenfa HUANG (Corresponding author) \\ E-mail: hshf3579@qztc.edu.cn \\ TSL Business School, Quanzhou Normal University \\ Professor Chun JIN, PhD \\ E-mail: jinchun@dlut.edu.cm \\ Institute of Systems Engineering, Dalian University of Technology
}

\title{
3D-CONTAINER LOADING PROBLEM WITH A DISTRIBUTION PLAN BASED ON HYBRID QUANTUM GENETIC ALGORITHM
}

\begin{abstract}
This paper proposes a multiobjective optimization model of a three dimensional container loading problem( $3 D-C L P)$ considering the distribution plan and designs its hybrid quantum genetic algorithm (HQGA). The purpose of the model is to maximize the rate of space utilization and to adjust the loading structure through simple parameter initialization. A hybrid algorithm is put forward based on a quantum genetic algorithm, and spaces depict strategy based on the surfaces. For this reason, the algorithm introduced in this paper proposed a new quantum coding pattern. Compared to the traditional evolutionary algorithm, the quantum genetic algorithm has more powerful capacity in traversal search. Finally, an actual numerical experiment and a comparison test were illustrated to verify the efficient and stable performance of the proposed algorithm. The results have shown that the solution has a satisfactory, stable, compact effect and an adjustable loading structure.
\end{abstract}

Keywords: $3 D$ container loading problem, Quantum genetic algorithm, Multi-objective optimization, Quantum coding, Loading structure.

\section{JEL Classification: C06, C61, C63}

\section{Introduction}

The loading problem is a complicated combination optimization. Research on the loading problem mainly focuses on its solution algorithm[1]. This mainly relates to the changes of packing constraints in the real world. Until now, there have been many research efforts devoted to it. Iwasawa et al proposed a heuristic algorithm based on a greedy strategy for the container loading problem with complex loading constraints[2]. Based on the concept of 'Block', Fanslau et al designed an orientated tree algorithm of efficient paths by stimulating the growth of the trees, which belongs to the heuristic algorithm[3]. Gonçalves et al designed a

DOI: 10.24818/18423264/55.4.21.08 
multigroup parallel and adaptive genetic algorithm based on the empty maximalspaces[4]. Liang et al proposed a hybrid meta-heuristic based on a two-phased method focusing on the improvement of the efficiency and on the reduction of the loading problem size[5].

Computing the complexity of traditional algorithms presents exponential growth with the growth of unloaded item quantity[6]. 3D-CLP is one of the most complicated loading problems. Currently, more and more researchers pay attention to 3D-CLP, with the increasing number and various shapes of items. Toffolo et al presented a decomposition approach embedded in a multiphase heuristic for the multiple container loading problem introduced by the Renault company in France. This approach requires a large number of different items to be packed into containers of different types and sizes[7]. Zhao et al built a multiobjective mixed integer programming model of the three-dimensional packing problem for solving 3D-CLP with eight kinds of practical constraints[8]. Leonardo et al presented heuristic algorithms based on the combination of two classic heuristics from vehicle routing and container loading problems to solve a three-dimensional loading capacitated vehicle routing problem[9]. Wu et al developed three-stage heuristic algorithms to solve a new practical three-dimensional packing problem that can be decomposed into three subproblems: three-dimensional irregular packing with variable-size cartons problem, three-dimensional variable-size bin packing problem, and the single container loading problem[10]. Lin et al established a model for the two-door 3D-CLP under home delivery service, which predetermines cargo delivery ordering and aims to reach maximal utilization ratio of the container space[11]. Zhao et al provided an extensive comparison of algorithm performance across the benchmark literature and focused on some of the critical algorithmic design issues[12]. Célia et al developed MIP-based constructive heuristics to quickly provide good initial and feasible solutions for the threedimensional multiple bin size bin packing problem[13]. Turky et al presented iterated local search heuristics with guided perturbation for the heterogeneous fleet vehicle routing problem with time windows and three-dimensional loading constraints that must be solved within one hour[14]. In addition, a genetic algorithm-applied multipopulation strategy and fuzzy logic controller (FLC) were proposed to improve the efficiency and effectiveness of the 3D container loading problem[15].

The above recent research on 3D-CLP has mainly focused on the simplex optimization method of the loading rate. The real logistic operation will adopt different loading plans to items due to different needs and simultaneously consider the safety of distribution. This approach demands that the total center of gravity of loaded items cannot be excessively offset in the axial direction. Thus, 3D-CLP modeling and solving become challenging operations in reality. There are substantially fewer correlation research efforts. Therefore, a 3D-CLP concerned with the distribution plan is proposed in this paper. First, the distribution plan for goods is ascertained, and then an adjustable loading structure plan is designed by 


\section{D-Container Loading Problem with a Distribution Plan Based on Hybrid Quantum Genetic Algorithm}

dividing the load space to make the solution plan satisfy the real needs. Next, an HQGA is designed, which combines QGA with the spatial description strategy based on planar considerations. Finally, the excellent global searching ability of QGA is used to avoid the local optimum. This paper is organized as follows. In Section 2, we describe the questions addressed. Section 3 presents the model of 3D-CLP with the distribution plan. The detailed algorithm is introduced in Section 4. Section 5 reports the computational results of the practical test instances. Finally, the last section summarizes the work.

\section{Question Description}

This paper primarily considers maximizing the space utilization. So the 3DCLP studied in this paper can be described as follows: given an ex-warehouse item sequence $\mathrm{C}$, loading space $\mathrm{R}$ and distribution plan, the objective of the solution is to load as many items as possible in a given loading space and to satisfy the requirements of the loading-unloading sequence and adjustable loading structure in terms of multi-destination distribution. Loading structure refers to the loading scheme formed by different occupancy sequences of items. Loading structure is represented by $S, S=[1,2,3]$, which means that goods are filled from bottom to top, from inside to outside and from side to side, respectively. Loading structure corresponds to placing flatwise, placing nearest the inside and placing by one side in the actual loading process. Assuming that a certain task needs to distribute $N$ items from $M$ species, these items need to be distributed to $P$ destinations. The problem statements are as follows:

(1) $R=[L, W, H, T, L B]$ denotes the loading space of the item. $L, W, H, T$ and $L B$ represent length, width, height, maximum load and the space load-bearing level on the bottom, respectively.

(2) $C=\left[c_{1}, c_{2}, \ldots, c_{1} \ldots, c_{N}\right]$ denotes the sequence of items, storing all items to be loaded. The attributes of each item $c_{\mathrm{i}}$ in the listing $C$ are represented by

$c_{\mathrm{i}}=\left[v_{i}, l_{i}, w_{i}, h_{i}, t_{i}, l b_{i}, x_{i}, y_{i}, z_{i}, e_{i}, d_{i}, g_{i}\right]$. The symbols in ci denote whether the item is loaded or not. They also denote the length, width, height, weight of the item, the load-bearing level, the coordinate position of the left rear point of the item, placement mode, the destination code of the item and the center of gravity of the item after loading, in which:

(1) The parameter $v_{\mathrm{i}}$ fetches a 0 or 1 value, which indicate that the item is unloaded or loaded, respectively.

(2) The load-bearing parameter $l b_{\mathrm{i}}$ is expressed by a positive integer. The larger the $\mathrm{lb}$ values, the greater the load-bearing level of the item is.

(3) Destination code $d_{\mathrm{i}}$ denotes the distribution location of items. The destination data are needed to solve the loading problems arising from the results of vehicle routing planning in the early stage of the logistics center. In this paper, $P$ destinations to be delivered are represented by destination set $D=[1,2,3 \ldots P], d_{\mathrm{i}} \in D$. 
The size of $d_{\mathrm{i}}$ indicates the priority level of distribution. The bigger the $d_{\mathrm{i}}$ is, the higher priority the item distribution will be. Correspondingly, the smaller the $d_{\mathrm{i}}$ is, the lower priority the current item loading is. Thus, this can meet the needs of loading-unloading operation. Therefore, $d_{\mathrm{i}}$ can also indicate the priority of item loading. The smaller the $d_{\mathrm{i}}$ is, the lower priority the loading will be.

(4) In this paper, we assume that the center of gravity of each item to be loaded is its geometric center, and the center of gravity of the item loaded is expressed by $g_{\mathrm{i}}, g_{\mathrm{i}}=\left(x_{\mathrm{i}}, y^{\prime}{ }_{\mathrm{i}}, z_{\mathrm{i}}{ }_{\mathrm{i}}\right)$.

(5) The placement mode parameter $e_{\mathrm{i}}$ fetches the value ranging from 1 to 6 . The placement mode represents the relationship between the unilateral dimension of the item and the coordinate axis, which is shown in Table 1 below.

Table 1. Mapping table of placement method $e i$

\begin{tabular}{|c|c|c|}
\hline$e_{i}$ & Parallel Relation of Item Unilateral Dimension and Axis & Placement Method \\
\hline 1 & $l_{i} / / X, \quad w_{i} / / Y, \quad h_{i} / / Z$ & Standing \\
\hline 2 & $l_{i} / / Y, \quad w_{i} / / X, \quad h_{i} / / Z$ & Standing-Rotating \\
\hline 3 & $l_{i} / / X, \quad w_{i} / / Z, \quad h_{i} / / Y$ & Lying \\
\hline 4 & $l_{i} / / Y, \quad w_{i} / / Z, \quad h_{i} / / X$ & Lying-Rotating \\
\hline 5 & $l_{i} / / Z, \quad w_{i} / / Y, \quad h_{i} / / X$ & Side Lying \\
\hline 6 & $\begin{array}{lll}l_{i} / / Z, & w_{i} / / X, & h_{i} / / Y\end{array}$ & Side Lying-Rotating \\
\hline
\end{tabular}

At the same time, this paper designs a description strategy of loading space based on a plane[6]. The plane refers to the bottom of the loading space and the surfaces of other objects. The paper describes the loading space by defining a plane list. The plane list is expressed as $P=\left[\mathrm{p}_{1}, p_{2}, \ldots p_{\mu}\right]$, where $\mu$ is the number of planes, $p_{\mathrm{i}} \in P$. The attributes of each plane pi are represented by $p_{\mathrm{i}}=\left[p l_{\mathrm{i}}, p w_{\mathrm{i}}, p h_{\mathrm{i}}, l b_{\mathrm{i}}, L w_{\mathrm{i}}, x_{\mathrm{i}}\right.$, $\left.y_{\mathrm{i}}, z_{\mathrm{i}}\right]$, and the coordinate axis is shown in Figure 1, in which:

(1) $p l_{\mathrm{i}}$ represents the length of the plane along the $\mathrm{X}$ direction in the coordinate axis. (2) $p w_{\mathrm{i}}$ represents the length of the plane along the $\mathrm{X}$ direction in the coordinate axis. (3) $p h_{\mathrm{i}}$ represents the length of the plane along the $\mathrm{Z}$ direction in the coordinate axis. (4) $l b_{\mathrm{i}}$ represents the load-bearing level of the plane, which is determined by the type of plane. (5) $x_{\mathrm{i}}, y_{\mathrm{i}}, z_{\mathrm{i}}$ represent the coordinates of the left rear point of the plane, which are in the same coordinate axis as the loaded items.(6) $L w_{\mathrm{i}}$ represents the maximum load limit of the plane. The maximum load of each plane in the loading process is updated in real time according to the loading situation. Thus, when the maximum load of a plane needs to be calculated, the maximum load of the loading space is used to subtract the total load of the loaded items. The loaded items can be determined according to the item sequence $C$. 
3D-Container Loading Problem with a Distribution Plan Based on Hybrid Quantum Genetic Algorithm

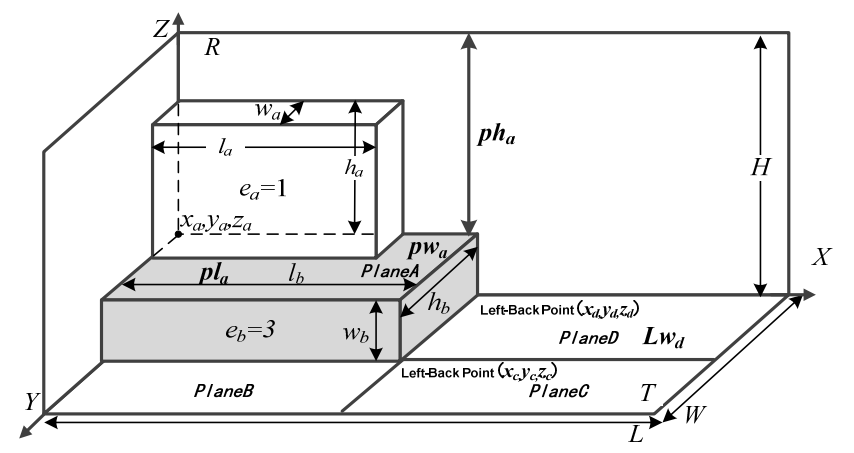

Figure 1. 3D-CLP problem schematic diagram

Plane list $P$ is at the bottom of the space in the initial situation. In the process of continuously placing items, the list is updated dynamically in real time. In this paper, the plane list records the plane of the items that have not been placed. After placing items on a certain plane, the original plane will be removed from the list and added to the new plane.

As shown in Figure 1, we set up a coordinate system to represent the loading of items. We describe the meaning of the parameters with the loaded items $c_{\mathrm{a}}, c_{\mathrm{b}}$, in which:

(1) Loading label $v_{\mathrm{a}}=v_{\mathrm{b}}=1$ indicated that two items $c_{\mathrm{a}}, c_{\mathrm{b}}$ are loaded into the loading space $R$; (2) Items $c_{\mathrm{a}}, c_{\mathrm{b}}$ satisfy $l b_{\mathrm{a}} \leq l b_{\mathrm{b}}$, that is, the load-bearing level of item $\mathrm{b}$ is greater than or equal to the load-bearing level of item $a$, so item ca can be placed on item $c_{\mathrm{b}}$. (3) The length, width, height and weight of items $c_{\mathrm{a}}, c_{\mathrm{b}}$ are marked in Figure 1. (4) The left rear coordinates of item $c_{\mathrm{b}}$ are $(0,0,0)$, and the left rear coordinates of item $c_{\mathrm{a}}$ are $\left(0,0, z_{\mathrm{b}}\right) .(5)$ The placement mode of item $c_{\mathrm{a}}$ is $e_{\mathrm{a}}=1$, which means that the item is placed vertically; the placement mode of item $c_{\mathrm{b}}$ is $e_{\mathrm{b}}=3$, which means that the item is placed flatwise.

\section{Model Construction}

In this paper, the description of the objective function with 3D-CLP is as follows:

obj.

$\max F_{1}=\sum_{i=1}^{N}\left(v_{i} \times l_{i} \times w_{i} \times h_{i}\right) /(L \times W \times H)$

$\min F_{2}=\sqrt{\left(y^{R}-W / 2\right)^{2}+\left(z^{R}-H / 2\right)^{2}}$

where, 
Yingyi Huang, Shenfa Huang

$$
\begin{aligned}
& y^{R}=\sum_{1}^{N} v_{i} \times y_{i}^{\prime} \times t_{i} / \sum_{1}^{N} v_{i} \times t_{i} \\
& z^{R}=\sum_{1}^{N} v_{i} \times z_{i}^{\prime} \times t_{i} / \sum_{1}^{N} v_{i} \times t_{i} \\
& \sum_{i=1}^{N}\left(v_{i} \times l_{i} \times w_{i} \times h_{i}\right) \leq L \times W \times H \\
& \sum_{i=1}^{N} v_{i} \times t_{i} \leq T \\
& e_{i}=1, x_{i}+l_{i} \leq L, y_{i}+w_{i} \leq W, z_{i}+h_{i} \leq H \\
& e_{i}=2, x_{i}+w_{i} \leq L, y_{i}+l_{i} \leq W, z_{i}+h_{i} \leq H \\
& e_{i}=3, x_{i}+l_{i} \leq L, y_{i}+h_{i} \leq W, z_{i}+w_{i} \leq H \\
& e_{i}=4, x_{i}+h_{i} \leq L, y_{i}+l_{i} \leq W, z_{i}+w_{i} \leq H \\
& e_{i}=5, x_{i}+h_{i} \leq L, y_{i}+w_{i} \leq W, z_{i}+l_{i} \leq H \\
& e_{i}=6, x_{i}+w_{i} \leq L, y_{i}+h_{i} \leq W, z_{i}+l_{i} \leq H \\
& z_{i}=\left\{\begin{array}{c}
z_{j}+h_{j} \quad e_{j} \in\{1,2\}, l b_{i} \leq l b_{j} \\
z_{j}+l_{j} \quad e_{j} \in\{3,4\}, l b_{i} \leq l b_{j} \\
z_{j}+w_{j} \quad e_{j} \in\{5,6\}, l b_{i} \leq l b_{j} \\
0 \quad \text { else }
\end{array} \quad \begin{array}{c}
\text { and } \quad L w>0 \\
L w=T-\sum_{1}^{k} t_{i} \times n_{i} \quad \text { and } j \in[1, N]
\end{array}\right.
\end{aligned}
$$

The objective function (1) shows that the optimization goal is maximizing the utilization of loading space. The objective function (2) presents the items' center weighted and minimum offset of loading space in the YZ directions; formulas (3-1) and (3-2) refer to the center weighted items in the $\mathrm{YZ}$ directions. When considering the general encasement problems, the objective function (2) is used first in this paper. While considering the loading problems, the high efficiency of truck loading should be required, and the axial distance migration of the center weight after loading the items should not be too large.

Formulas (4) to (8) are the constraint conditions of 3D-CLP. Constraint condition (4) refers to the situation that the sum of the loading item total volume is less than the loading space; constraint condition (5) shows that the sum of item weight is less than the maximum load of the loading space; in the constraint condition (6), at least one can be guaranteed to ensure that the item ci to be placed does not exceed the limitations of the length, width and height of the loading space; constraint condition (7) states that the cargo in the upper layer must be supported by the cargo in the lower layer: the cargo cannot be hung and must satisfy the loadbearing level constraints of items when stacked; constraint condition (8) is the maximum load solution of the single plane in its list and must be greater than zero, in 


\section{D-Container Loading Problem with a Distribution Plan Based on Hybrid}

Quantum Genetic Algorithm

which case the symbols refers to k kinds of loaded items at some moment, $\mathrm{n}$ quantity of each loaded item, and ti weight of the item itself.

\section{Algorithm Design}

In this paper, a heuristic strategy is used as a decoding mapping function to calculate fitness. As a new arithmetical method, QGA has more powerful capacity in traversal searching, and it does not readily fall into local optimum and fast convergence to the global optimum solution with small population size compared with traditional GA. At present, there are few studies on quantum coding methods for 3D-CLP. A new quantum chromosome coding method is proposed, and the corresponding population initialization method is introduced.

\subsection{Quantum Coding Design}

We use quantum bit chromosomes to code the three-dimensional packing problem space and propose a combined coding design method, as shown in Figure 2 below. Each chromosome Gen consists of IFLoaded, Mode, and Put, which are described below.

\begin{tabular}{|c|c|c|c|c|c|c|c|c|}
\hline \multicolumn{3}{|c|}{ IFLoaded } & \multicolumn{4}{|c|}{$\underbrace{\text { Mode }}$} & \multicolumn{2}{|l|}{$\underbrace{P u t}$} \\
\hline & & $\ldots \quad a_{N}$ & $m_{1}$ & & $\cdots$ & $m_{M}$ & $p_{1}^{1}, p_{2}^{1}, p_{3}^{1}, p_{4}^{1}, p_{5}^{1}, p_{6}^{1}$ & $\ldots p_{4}^{M}, p_{5}^{M}, p_{6}^{M}$ \\
\hline$b$ & $b_{2}$ & $\ldots b_{N}$ & $n_{1}$ & $n_{2}$ & $\cdots$ & $n_{M}$ & $q_{1}^{1}, q_{2}^{1}, q_{3}^{1}, q_{4}^{1}, q_{5}^{1}, q_{6}^{1}$ & $\ldots q_{4}^{M}, q_{5}^{M}, q_{6}^{M}$ \\
\hline
\end{tabular}

Figure 2. Structure of quantum bits code chromosome

(1) Whether the item is loaded in the sequence, IFLoaded=[ifload 1 , ifload $_{2}, \ldots$,ifload $\left.{ }_{\mathrm{N}}\right]$. In ifloadi $=\left[a_{\mathrm{i}}, b_{\mathrm{i}}\right] \mathrm{T}$, natural number $1,2, \ldots, \mathrm{N}$ denotes the number of items to be loaded, $a_{\mathrm{i}}$ denotes the probability that item $i$ is loaded, and $b_{\mathrm{i}}$ denotes the probability that item $i$ is unloaded.

(2) Placement sequence Mode $=[\operatorname{mode} 1, \operatorname{mode} 2, \ldots, \operatorname{modeM}]$. In modei $=\left[m_{\mathrm{i}}, n_{\mathrm{i}}\right]^{\mathrm{T}}$, natural number $1,2, \ldots \mathrm{M}$ denotes the number of items to be loaded.

(3) The sequence of placement of items: Put $=\left[\right.$ put $_{1}$, put $_{2}, \ldots$, put $\left._{M}\right]$. In this paper, there are six ways to place objects, including standing lay, standing and spin lay, lying, lying \& spin, side lay, side \& spin lay, and the placement direction of items $\mathrm{h}$ parallel or vertically to the $x, y, z$ axis. In addition, $\operatorname{put}_{\mathrm{i}}=\left[p^{\mathrm{i}}, q^{\mathrm{i}}\right]^{\mathrm{T}}, p^{\mathrm{i}}=\left[p_{1}, p_{2}, p_{3}, p_{4}, p_{5}, p_{6}\right]^{\mathrm{T}}$ is the probability of the first item's permissible placement mode. $q^{\mathrm{i}}=\left[q_{1}, q_{2}, q_{3}, q_{4}, q_{5}, q_{6}\right]^{\mathrm{T}}$ is the probability of the first item's unallowed placement mode.

Thus, any loading of the items to be loaded can be represented by chromosome Gen $=\{$ IFLoaded, Mode, Put $\}$. The data structure of the encoding space is shown in Figure 3. Each chromosome describes whether each item in the list is loaded, paved 
or stacked, as well as the placement allowed. According to the method of loading and the property of the item determined by the chromosome, the decoding strategy is designed to map the decoding of the chromosome structure to the loading space to determine the loading scheme.

\begin{tabular}{|c|c|c|}
\hline $\begin{array}{c}\text { Data Structure Of } \\
\text { Quantum Chromosomes }\end{array}$ & \multicolumn{1}{c|}{$\begin{array}{c}\text { Data Structure Of } \\
\text { Item Sequence }\end{array}$} \\
\hline IFLoadedSequence & & 3-dimensional size: $l, w, h$ \\
\hline ModeSequence & & $\begin{array}{l}\text { Weight and grade: } t, l b \\
\text { point: } x, y, z\end{array}$ \\
\hline PutSequence & Loading number: $N S$ \\
\hline List of enabled placing ways: $A[6]$ \\
\hline
\end{tabular}

Figure 3. Data structure of a chromosome and item sequence

\subsection{Initialization}

Initialization of quantum chromosomes adopts the method of placing the probabilistic amplitude of the locus at $1 / \sqrt{2}$.. This method indicates that in the primary chromosomes, each locus of each chromosome is observed with the same probability of $1 / 2$, that is, each locus represents an equal probability superposition of all possible states.

The population $Q(\mathrm{t})$ must be observed after the initialization of the quantum population. The paper uses the random numbers generating method to measure the population, and the results are shown as a binary solution $P(\mathrm{t})$. The observation process of quantum chromosomes for a three-dimensional packing problem is shown in Figure 4, including two kinds of item' number 2.

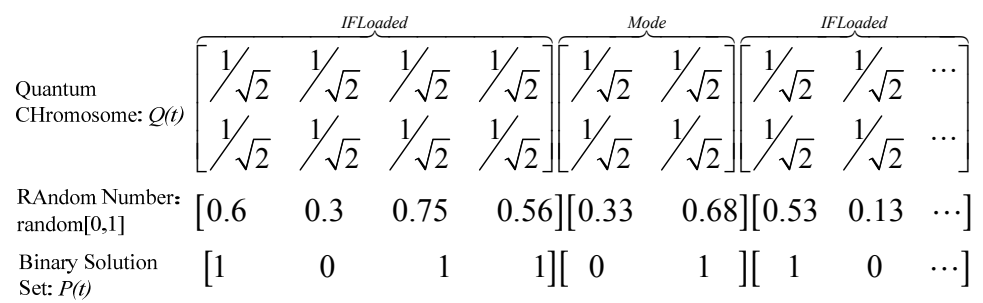

Figure 4. Observation process of quantum chromosomes

\subsection{Decoding Strategy}

The decoding strategy is based on the plane in the loading space. The paper gives more attributes to the plane. The above strategy can track the loading 


\section{D-Container Loading Problem with a Distribution Plan Based on Hybrid Quantum Genetic Algorithm}

sequence well and record the loading situation of the space. The core idea of the decoding strategy is to describe the space loading situation with the use of a flat list. According to $P(\mathrm{t})$, a list of items to be loaded can be determined. The attributes of each item in the list are determined by the data structure shown in Figure 3.

\subsubsection{Plane choice and item filling}

When filling an item, first select the plane in the list of planes. The plane selection strategy determines the structure of the loading scheme. According to the properties of the plane, three loading structures are designed in this paper: flat, inward and sideward.

For the selected current plane, $P(\mathrm{t})$ determines the object placement and placement attributes for filling items. For the placing mode of flat pavement, the method of occupancy rate of the flat area is used to select the goods with the greatest occupancy rate of the current flat area. For the stacking mode, the goods with the greatest occupancy rate are selected, as shown in formula (9):

$\min H \% l$

in which $l$ ' indicates the height of an object in a certain placing mode.

\subsubsection{Initialization and update of plane list}

(1) Initialization of plane list

Initially, the unloaded loading space contains only one plane, $\mathrm{P}=[p]$, where $p=[L, \boldsymbol{W}, \boldsymbol{H}, \boldsymbol{L} \boldsymbol{B}, \boldsymbol{T}, 0,0,0]$. In this paper, the loading space is initialized by dividing the bottom $\mathrm{G}$ of the loading space equally, $\mathrm{G}=\mathrm{P}$, that is, the partition parameter of the loading space is the number of distribution destinations. This paper adopt two methods of division:

(1) Horizontal division. Division according to the $\mathrm{X}$-axis direction of loading space. Assume that there are 3 distribution destinations in the plane. $\mathrm{P}=\left[p_{1}, p_{2}, p_{3}\right]$ is the flat list of loading space, where $p_{1}=\left[L / 3, W, H, L B, T_{1}, 0,0,0\right], \quad p_{2}$ $=\left[L / 3, W, H, L B, T_{2}, L / 3,0,0\right]$, and $p_{3}=\left[L, W, H, L B, T_{3}, 2 L / 3,0,0\right]$.

(2) Vertical division. Division according to the $\mathrm{Y}$-axis direction of loading space. The flat list of loading space is initialized to $\mathrm{P}=\left[p_{1}, p_{2}\right]$, where $\mathrm{p} 1=p_{1}$ $=\left[L, W / 2, H, L B, T_{1}, 0,0,0\right]$, and $\mathrm{p} 2=\left[L, W / 2, H, L B, T_{2}, 0, W / 2,0\right]$.

(2) Update of plane list

For the current plane, the current operating plane is divided into four parts as shown in Figure 2. In this paper, the principle of keeping as large a plane as possible, that is, satisfying the situation of area $\mathrm{S}(\mathrm{A})+\mathrm{S}(\mathrm{B})>\mathrm{S}(\mathrm{B})+\mathrm{S}(\mathrm{C})$, is adopted. Therefore, the current plane is divided into three planes: the upper surface of the object, Plane A and Plane $(\mathrm{B}+\mathrm{C})$. The updating strategy of the plane list is as follows: after a plane in the list is determined to be able to be placed in the items to be loaded, the current plane is deleted from the plane list, and the newly generated three planes are added to the plane list. 


\subsubsection{Decoding process}

Take two kinds of items as examples, each containing two quantum chromosomes. Assume that $\mathrm{A}=\{[1,0,1,1][0,1][1,0,1,1,1,1,1,1,1,0,0,0]\}$ is the binary solution of quantum chromosomes, and the corresponding decode forward of $\mathrm{A}$ is shown as follows.

(1) Place the flat item of the 1st kind of items, no standing-rotating.

(2) Stack two of the 2nd kind of items: no lying-rotating, no side lying, no side lying-rotating.

(3) As shown in Figure 5(a), place the 1st kind of item flat, while the current flat list is initialized, and the standing space can reach maximum occupancy. Also shown in Figure 5(b), stack the 2nd kind of items, and the lying method can reach maximum height. Choose PlaneB for lying once PlaneA and PlaneC cannot be placed. Arrows represent the directions of items.

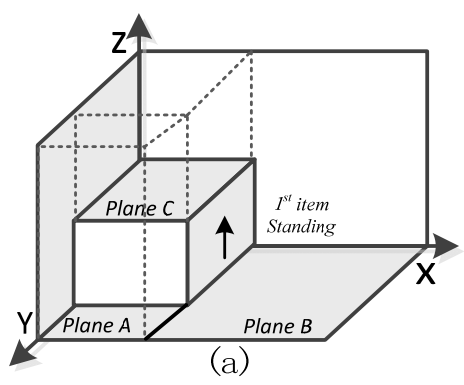

Figure 5. Decoding of chromosomes A

\subsection{Correction Strategy}

There may be chromosomes that do not meet the constraints after decoding into a binary solution, such as when the sum volume of items exceeds the total volume of load-space or the sum weight of items exceeds the total weight of loadspace. This will cause the chromosomes to be unable to reach a feasible solution. This paper used the following correction strategy.

(1) IFLoaded list correction. When the sum volume of items exceeds the total volume of load-space, the paper uses a correction strategy: the strategy randomly selects a location in the IFLoaded list and sets the value to " 0 ". Furthermore, for fear of overloading the items, the strategy randomly selects a location in the IFLoaded list and sets the value to "1", while the sum volume of items does not exceed the total volume of load-space. If the constraints are met, keep the results; if unsatisfied, then revert the value to " 0 ". The core concept of the correction strategy is to help to select items as much as possible on the condition that the sum weight and volume of items do not exceed the limit. 
3D-Container Loading Problem with a Distribution Plan Based on Hybrid Quantum Genetic Algorithm

(2) Put array correction. The decoding results of the Put array stipulate the place method for an item. To gain as much lying mode as possible, a gene value of 0 gain decoding from the binary solution $P(\mathrm{t})$ mutates into 1 by using random mutation in the paper.

\subsection{Fitness Function Design}

The design of a fitness function is based on the objective function of the paper's model, just as shown in formula (10)

$$
\max F^{f i t}=\xi_{1} \times F_{1}^{\prime}+\xi_{2} \times F_{2}^{\prime}
$$

where, the weight coefficient $\xi_{1}$ and $\xi_{2}$ are assigned due to the object's importance.

This converts $F_{1} 、 F_{2}$ to maximum $F_{1}$ '、 $F_{2}$ ' individually and uses $[0,1]$ to

standardize $F_{2}^{\prime}$.In addition, $\xi_{1}+\xi_{2}=1$.

$$
\begin{gathered}
F_{1}^{\prime}=F_{1} \\
F_{2}^{\prime}=1-F_{2} / \sqrt{\frac{L^{2}+W^{2}+H^{2}}{4}}
\end{gathered}
$$

Formula (11) is expressed as the maximum utilization rate of loading space. Formula (12) is represented as the shift extent of the weighted center after loading. The distance from geometric center to the distal apex in loading space is the maximum shift extent. The smaller the shift extent of the weighted center F3 is, the greater F'3. The paper designed different fitness functions to match with and solve two kinds of problems, including items and truck loading problems of the logistics center.

(1) The maximum utilization rate is the main objective of the item loading problem. It has no special demand for the core of the loading plan.

(2) In addition to the maximum utilization rate, the distribution between many destinations is also involved. Thus, two targets must be considered simultaneously: note that the shift extent between the weighted center of the loading plan and the central axis of the transport volume is not too large.

\subsection{Updating Mechanism}

The quantum rotation gate can rotate the phase of single-qubit to an angle to update the quantum chromosomes. The updating mechanism idea of the quantum rotation gate is to make the current solution converge quickly to a chromosome with better fitness. The updating process of $\left(\alpha_{k}, \beta_{k}\right)$, with the $k$ th qubit located in the quantum chromosomes, is shown as formula (13) and formula (14).

$$
\begin{gathered}
{\left[\begin{array}{l}
\tilde{\alpha}_{k} \\
\tilde{\beta}_{k}
\end{array}\right]=\left[\begin{array}{cc}
\cos \theta_{k} & -\sin \theta_{k} \\
\sin \theta_{k} & \cos \theta_{k}
\end{array}\right]\left[\begin{array}{l}
\alpha_{k} \\
\beta_{k}
\end{array}\right]} \\
\theta_{k}=s\left(\alpha_{k} \beta_{k}\right) \Delta \theta_{k}
\end{gathered}
$$


Yingyi Huang, Shenfa Huang

where $\Delta \theta_{\mathrm{k}}$ is the rotation angle of the quantum rotation gate, and $S\left(\alpha_{k} \beta_{k}\right)$ decides the direction that converges to the global optimization result. The choice of $S\left(\alpha_{k} \beta_{k}\right)$ and $\Delta \theta_{\mathrm{k}}$ is a convergence strategy, and their values are as shown in Table 2 . $b_{\mathrm{k}}$ and $x_{\mathrm{k}}$ represent the optimal solution of current population and the $k$ th value of the current solution set, respectively. $f(\mathrm{x})$ and $f(\mathrm{~b})$ are the corresponding objective functions. The convergence strategy used in the paper is shown as follows: in any case, the current solution with better fitness is an optimum solution. Furthermore, to avoid the premature convergence, one strategy was to adopt a slower convergence speed when the current solution equals the $k$ th value of the current solution set; in contrast, a faster convergence speed is used, while the two are not equal.

Table 2. Lookup table of $\Delta \theta_{\mathrm{k}}$

\begin{tabular}{|c|c|c|c|c|c|c|c|}
\hline & \multirow{2}{*}{$x_{\mathrm{k}}$} & $b_{\mathrm{k}}$ & \multirow{2}{*}{$f(\mathrm{x}) \geq f(\mathrm{~b})$} & \multicolumn{5}{|c|}{$s\left(\alpha_{\mathrm{k}} \beta_{\mathrm{k}}\right)$} & \multirow{2}{*}{$\Delta \theta_{\mathrm{k}}$} \\
\cline { 4 - 7 } & & $\alpha_{\mathrm{k}} \beta_{\mathrm{k}}>0$ & $\alpha_{\mathrm{k}} \beta_{\mathrm{k}}<0$ & $\alpha_{\mathrm{k}}=0$ & $\beta_{\mathrm{k}}=0$ & \\
\hline 0 & 0 & False & -1 & +1 & \pm 1 & 0 & $0.05 \pi$ \\
\hline 0 & 0 & True & -1 & +1 & \pm 1 & 0 & $0.05 \pi$ \\
\hline 0 & 1 & False & +1 & -1 & 0 & \pm 1 & $0.2 \pi$ \\
\hline 0 & 1 & True & -1 & +1 & \pm 1 & 0 & $0.4 \pi$ \\
\hline 1 & 0 & False & -1 & +1 & \pm 1 & 0 & $0.2 \pi$ \\
\hline 1 & 0 & True & +1 & -1 & 0 & \pm 1 & $0.4 \pi$ \\
\hline 1 & 1 & False & +1 & -1 & 0 & \pm 1 & $0.05 \pi$ \\
\hline 1 & 1 & True & +1 & -1 & 0 & \pm 1 & $0.05 \pi$ \\
\hline
\end{tabular}

\section{Experimental study}

The experimental environment of the algorithm is as follows: CPU: Intel(R) Core(TM)2 Duo E7400@2.80 GHz; memory: 2.00 GB; algorithm achieved using $\mathrm{CH}$. The basic parameter selection of the algorithm is shown as follows: population size NIND $=10$, iterative times MAXGEN $=300$.

\subsection{Testing the adjustable characteristic of loading structure}

In this paper, we propose that the loading structure plan must satisfy the requirement of loading structure to adapt to real encasement needs. The data resulting from testing 6 kinds of 600 encasement item problems, when the weight coefficients are set to $\xi_{1}=0.8$ and $\xi_{2}=0.2$, are shown in Table 3 .

Table 3. Example test data

\begin{tabular}{|c|c|c|c|c|c|c|c|}
\hline Item & $\begin{array}{c}\text { Length } \\
(\mathrm{mm})\end{array}$ & $\begin{array}{c}\text { Width } \\
(\mathrm{mm})\end{array}$ & $\begin{array}{c}\text { Height } \\
(\mathrm{mm})\end{array}$ & Quantity & $\begin{array}{c}\text { Load } \\
\text { Level }\end{array}$ & $\begin{array}{c}\text { ItemAllowed } \\
\text { Placement } \\
\text { Direction }\end{array}$ & $\begin{array}{c}\text { Weight/Maximum } \\
\text { Load (kg) }\end{array}$ \\
\hline Container & 12912 & 2340 & 3100 & 1 & - & - & 28280 \\
\hline Cargo1 & 1500 & 500 & 500 & 100 & 1 & 6 & 95 \\
\hline
\end{tabular}


3D-Container Loading Problem with a Distribution Plan Based on Hybrid Quantum Genetic Algorithm

\begin{tabular}{|l|l|l|l|l|l|l|l|}
\hline Cargo2 & 720 & 310 & 560 & 100 & 1 & 6 & 12 \\
\hline Cargo3 & 550 & 440 & 660 & 100 & 1 & 6 & 28 \\
\hline Cargo4 & 430 & 410 & 450 & 100 & 2 & 6 & 36 \\
\hline Cargo5 & 720 & 310 & 560 & 100 & 2 & 6 & 15 \\
\hline Cargo6 & 610 & 350 & 500 & 100 & 3 & 6 & 24 \\
\hline
\end{tabular}

There are 3 types of loading structures acquired by calculating the data in Table 3.

(1) $S=1$ Flush system. This refers to the situation in which items occupy the loading space in the bottom preferentially, and items are loaded from the bottom to the top, layer upon layer.

(2) $\mathrm{S}=2$ Place inside. This refers to the situation in which items occupy the loading space in the inside preferentially, and items are loaded from the inside to the outside, with priority assigned to the high place.

(3) $\mathrm{S}=3$ Place in the side of space. This refers to the situation in which items are placed in the side of the space and occupy the side space preferentially.

\subsection{Distribution Plan Testing}

The truck in the problem is adapted to the 3D container loading problem, which is concerned with the distribution plan, in which items are delivered to various destinations according to the distribution plan, not only considering satisfying the maximization of the loading spatial utilization but also ensuring the gross center of gravity to be in the transport vehicle shaft. Table 3 is also used in testing data. It is convenient to analyze the loading plan results by changing the quantity of each item into 60 and loading items using all of the loading space. At this time, the weight coefficient $\xi_{1}$ has no influence on the result. Then, we test the weight coefficient $\xi_{2}$ with different values. The loading results under different parameters are shown in Figure 6, in which the dotted line represents the separation of the loading space, $G$ is the number of delivery destination-initialization parameters of the loading space, and $\mathrm{S}$ is the loading structure:

(1) Setting $\xi_{2}=0.5$ and $S=1$, spatial initialization is a lateral division. The algorithm optimizes the loading in each section. Although the weighted center of loading result is around the loading space geometric center, and the number of sections increases with the growth of $G$ value due to the larger weight of the target center of gravity offset, the effectiveness of loading results in this plan can still be guaranteed.

(2) Setting $\xi_{2}=0$ and $S=3$, spatial initialization is a vertical division. Due to not considering the weight of the target center of gravity offset, the weighted center of the loading result can be biased toward one side of the space so that items can be loaded from one side of the space, and at the same time, items in different sections will not superimposed on each other after adopting spatial initialization. 

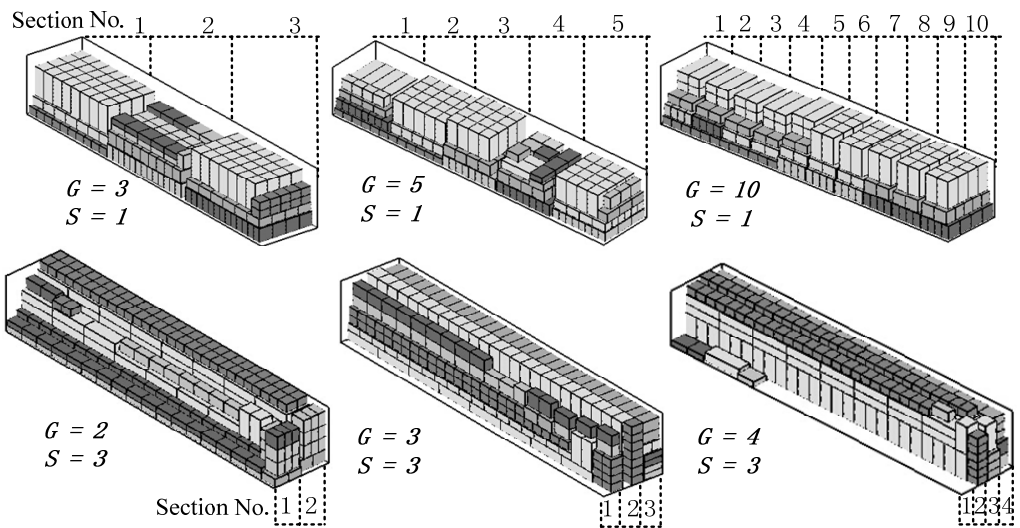

Figure 6. The Plan of 3D Container Loading Problem Considering the Distribution Plan and Load Configuration

\subsection{Loading Rate Testing}

The paper also used the data of references[16] and references[17] for testing. The testing results were shown in Table 4.

Table 4. Comparison of tests of loading rate

\begin{tabular}{|c|c|c|c|c|c|}
\hline Number & Example & $\begin{array}{c}\text { Item } \\
\text { Type }\end{array}$ & $\begin{array}{c}\text { Total } \\
\text { Number } \\
\text { of Items }\end{array}$ & $\begin{array}{c}\text { Loading Rate } \\
\text { in } \\
\text { Original } \\
\text { Literature }\end{array}$ & $\begin{array}{c}\text { Loading Rate } \\
\text { Obtained } \\
\text { in This Paper }\end{array}$ \\
\hline 1 & Literature[16] & 8 & 709 & $91.9 \%$ & $92.39 \%$ \\
\hline 2 & \multirow{2}{*}{ Literature[17] } & 5 & 1728 & $97.46 \%$ & $98.33 \%$ \\
\cline { 3 - 6 } & 11 & 637 & $92.40 \%$ & $93.45 \%$ \\
\hline
\end{tabular}

From the analysis of the test results, the loading rate of this algorithm is better for the packing problem of a large number of items. For fewer items and diverse types, the space utilization rate of this algorithm is not good, especially for the strong heterogeneous packing problem, which requires further optimization. Because of the distribution plan of the logistics center, this algorithm can effectively solve the actual packing problem.

\section{Conclusion and discussion}

In this paper, considering the distribution scheduling for the 3D container loading problem, the adjustable structure and its hybrid quantum genetic algorithm are proposed, and the main conclusions are as follows. (1) For the needs closely 
3D-Container Loading Problem with a Distribution Plan Based on Hybrid Quantum Genetic Algorithm

related to "loading" and "distribution" in real operation, in this paper, we construct a multiobjective optimization model of the container loading problem, which considers the distribution plan; based on the distribution scheduling to design the loading plan, we make the loading plan satisfy various constraints and multidestination requirements, as well as adjustable characteristics of loading structure. (2) In the algorithm design, the hybrid quantum genetic algorithm is put forward in this paper. Moreover, for the 3D container loading problem, a quantum coding pattern, decoding strategy and space depiction strategy based on the surfaces are designed. This algorithm achieves the adjustable characteristics of loading structure and excellent loading rate. (3)The results tested with instances show that this mode and algorithm can achieve the adjustable characteristics of the loading structure of the solution. Furthermore, the loading plan can satisfy the item partitioning when we consider the multi-destination distribution, and it has a better operation effect for the large-scale loading problems, which is able to maximize the rate of space utilization. This research provides new thought and an effective path to solve the 3D container loading optimization problem and encasement and loading problems in the logistics center.

\section{REFERENCES}

[1] Asit B., Dilip R.(2012), A Heuristic Modelling for Three-Dimensional Container Loading Problem. International Journal of Modelling in Operations Management, 2, 345-359;

[2] Iwasawa H., Hu Y.N., Hashimmoto H. , Imahori S. , Yagiura M.(2016), $A$

Heuristic Algorithm for the Container Loading Problem with Complex Loading Constraints. Journal of Advanced Manufacturing Systems, 10,1-12;

[3] Fanslau T., Bortfeldt A.(2010), A Tree Search Algorithm for Solving the Container Loading Problem. INFORMS Journal on Computing, 22, 222-235;

[4] Gonçalves J.F., Resende M.G.C.(2012), A Parallel Multi-Population Biased Random-Key Genetic Algorithm for a Container Loading Problem. Computers \& Operations Research, 39,179-190;

[5] Liang S.C., Lee C.Y., Huang S.W.(2014), A Hybrid Meta-Heuristic for the Container Loading Problem. Communications of the IIMA, 7, 72-84;

[6] Pisinger D.(2002), Heuristics for the Container Loading Problem. European Journal of Operational Research, 141,382-392;

[7] Toffolo T.A.M., Esprit E., Wauters T., Berghe G.V.(2017), A Two-

Dimensional Heuristic Decomposition Approach to a Three-Dimensional

Multiple Container Loading Problem. European Journal of Operational

Research, 257, 526-538; 
Yingyi Huang, Shenfa Huang

[8] Zhao N., Cui X.L., Han Q.W.(2017), An Optimization Algorithm for Solving Three-Dimensional Container Loading Problem with Practical Constraints. Industrial Engineering and Management, 22,10-16;

[9] Leonardo J. , Reinaldo M. (2015), Heuristic Algorithms for a ThreeDimensional Loading Capacitated Vehicle Routing Problem in a Carrier. Computers \& Industrial Engineering, 88,110-130;

[10] Wu H.T., Leung S.C.H., Si Y.W., Zhang D., Lin A.(2017), Three-Stage

Heuristic Algorithm for Three-Dimensional Irregular Packing Problem. Applied Mathematical Modelling, 41, 431-444;

[11] Lin C.C. , Kang J.R., Liu W.Y., Li C.C.(2016), On Two-Door Three-

Dimensional Container Packing Problem under Home Delivery Service. Journal

of Industrial and Production Engineering, 33,205-214;

[12] Zhao X.Z., Bennell J.A., Bektas T., Dowsland K.(2016), A Comparative

Review of 3D Container Loading Algorithms. International Transactions in Operational Research, 23, 287-320;

[13] Paquay C., Limbourg S., Schyns Michal., Oliveira J.F.(2018), MIP-Based Constructive Heuristics for the Three-Dimensional Bin Packing Problem with

Transportation Constraints. International Journal of Production Research, 56,1581-1592;

[14] Turky A. , Moser I. , Aleti A. (2017), An Iterated Local Search with Guided Perturbation for the Heterogeneous Fleet Vehicle Routing Problem with Time

Windows and Three-Dimensional Loading Constraints. Artificial Life and

Computational Intelligence, 10142,279-290;

[15] Zheng J.N., Chien C.F., Gen M. (2015), Multi-Objective Multi-Population

Biased Random-Key Genetic Algorithm for the 3-D Container Loading Problem. Computers \& Industrial Engineering, 89, 80-87;

[16] Zhai Y., Sun X.M. (2007), A Heuristic Algorithm for Three-Dimensional Container Loading Problem with Non-Identical Items. Journal of Shanghai

Jiaotong University, 41,1244-1247;

[17] Zhao Z.K., Mei G.J., Li H.G. (2013), Solution of 3D Packing Problem

Based on Heuristic Personified Algorithm. Logistics Technology, 32,252-254. 